\title{
Analysis of Spontaneous MEG Activity in Patients with Alzheimer's Disease using Spectral Entropies
}

\author{
Jesús Poza*, Student Member, IEEE, Roberto Hornero, Member, IEEE, Daniel Abásolo, Member, \\ IEEE, Alberto Fernández, and Javier Escudero, Student Member, IEEE
}

\begin{abstract}
The aim of this study was to explore the ability of several spectral entropies to discriminate between spontaneous magnetoencephalographic (MEG) oscillations from 20 Alzheimer's disease (AD) patients and 21 controls. Hence, the relative spectral power $(R S P)$ in classical frequency bands was calculated from the averaged power spectral density. Given the fact that the $R S P$ can be viewed as a probability distribution function, the Shannon spectral entropy, Tsallis spectral entropy, generalized escort-Tsallis spectral entropy and Rényi spectral entropy were calculated from the RSP. Significant differences for each parameter were assessed with MannWhitney $U$ test, whereas classification performance was studied using binary logistic regression. Results revealed an increase in the $R S P$ of control subjects at beta and gamma bands, while AD patients showed an increase in the RSP values at delta and theta bands. Entropic quantifiers obtained statistically significant lower values for AD patients than for controls. This issue suggests a significant decrease in irregularity of AD patients' MEG activity.
\end{abstract}

\section{INTRODUCTION}

A LZHEIMER'S disease (AD) is a primary degenerative dementia that gradually destroys brain cells and leads to progressive decline in mental function [1]. Differential diagnosis of $\mathrm{AD}$ is based on a complete medical evaluation. Nevertheless, the accuracy of clinical diagnosis is about $90 \%$ [2]. It can be confirmed only by histological examination of brain tissue.

In order to improve the diagnosis, electroencephalographic (EEG) brain activity has been widely analyzed. More recently, special attention has also been paid to magnetoencephalographic (MEG) activity. Both recordings are generated by synchronous oscillations of pyramidal neurons. However, EEG is sensitive to all primary currents, while MEG is insensitive to current flows oriented perpendicularly to the scalp. Since EEG and MEG detect slightly different features of the electromagnetic brain activity, they can be viewed as complementary techniques [3]. An important advantage of MEG over EEG arises from

Manuscript received April 13, 2007. This work was supported in part by the grant project VA108A06 from "Consejería de Educación de Castilla y León" and by the "Ministerio de Educación y Ciencia" and FEDER grant MTM2005-08519-C02-01. Asterisk indicates corresponding author.

J. Poza*, R. Hornero, D. Abásolo, and J. Escudero are with the Biomedical Engineering Group (GIB), Department T.S.C.I.T., University of Valladolid, Camino del Cementerio s/n, 47011-Valladolid, Spain (phone: +34 983 423000, ext. 5569; fax: +34 983 423667; e-mail: \{jesus.poza, roberto.hornero, daniel.abasolo\}@ tel.uva.es, javier.escudero@ieee.org).

A. Fernández is with the Centro de Magnetoencefalografía Dr. PérezModrego, Complutense University of Madrid, 28040-Madrid, Spain (e-mail: aferlucas@med.ucm.es). the insensitivity of magnetic fields to inhomogeneities in the head. This implies that MEG recordings are less distorted than EEG signals on the scalp [3].

Some abnormalities in moderate and severe AD patients' EEG and MEG background activity have been observed [4], [5]. Whereas the EEG has been broadly investigated using spectral and non-linear measures, only a few studies have analyzed the MEG patterns in AD. In this way, spontaneous MEG activity shows increased slow rhythms and reduced fast activity in $\mathrm{AD}$ patients in comparison with healthy subjects [6]-[8]. This fact has also been observed using some spectral parameters like mean frequency [9], [10] and individual alpha peak [7], [10], [11]. From another point of view, studies analyzing MEG recordings at rest showed a global decrease in irregularity [10] and complexity [12] when $\mathrm{AD}$ patients are compared with healthy controls. However, some authors only reported the loss of complexity in the high frequencies [13]. Other approaches, which focused on analyzing the functional connections rather than local abnormalities in $\mathrm{AD}$, have found both a decrease of coherence values in the alpha band [14] and a general decrease of coherence in all frequency bands [6]. A lower level of synchronization has also been reported in the upper alpha, beta and gamma bands of AD patients in comparison with controls, suggesting a loss of functional connectivity [15]. Recently, it has been observed that resting-state functional connectivity in $\mathrm{AD}$ is characterized by specific changes of long and short distance interactions in several frequency bands [16]. Therefore, it can be inferred that AD is related with an abnormal function of the large scale brain networks [16].

In this scenario, where highly nonlinear and long-range (for interneural distances) phenomena are present, a nonextensive analysis of the problem may be appropriate. In a similar context, previous studies have successfully applied several quantifiers based on extensive (Shannon) and nonextensive (Tsallis, generalized escort-Tsallis and Rényi) information measures to analyze EEG signals [17]-[20]. These non-extensive measures are controlled by the parameter $q$, which is modified to emphasize particular characteristics of the associated dynamics.

This study is a first approach to explore the ability of several measures from information theory to characterize MEG rhythms in AD. The proposed parameters are entropies based on the relative power distribution. Firstly, the Fourier transform (FT) was used to compute the power spectral density (PSD) for each MEG recording from AD patients and controls. Relative spectral power (RSP), Shannon spectral entropy ( $S S E)$, Tsallis spectral entropy 
(TSE), generalized escort-Tsallis spectral entropy (GSE) and Rényi spectral entropy ( $R S E)$ were then calculated. The $R S P$ was used to characterize the power distribution over the frequency and to confirm the MEG slowing in AD. On the other hand, the SSE was used to analyze the flatness of the spectrum, while the quantifiers based on non-extensive information measures (TSE, GSE and RSE) were employed to explore the irregularity of the recordings, modifying the distribution of the original signal.

\section{MAterials AND METHODS}

\section{A. Subjects and MEG recording}

Twenty patients $(7$ men and 13 women, age $=73.05 \pm$ 8.65 years, mean \pm standard deviation SD) from the "Asociación de Familiares de Enfermos de Alzheimer" (AFAL) were included in the study. All of them fulfilled criteria for probable AD according to the clinical guidelines of the National Institute of Neurological and Communicative Disorders and Stroke and the AD and Related Disorders Association (NINCDS-ADRDA) [21]. Their cognitive function was assessed using the Mini-Mental State Examination (MMSE), obtaining a mean MMSE score of $17.85 \pm 3.91$ (mean \pm SD). Moreover, AD patients were not taking any medication that could affect the central nervous system. Twenty-one cognitively normal volunteers (9 men and 12 women, age $=70.29 \pm 7.07$ years, mean \pm SD) participated in the study as controls. Their mean MMSE score was of $29.10 \pm 1.00$ points. No significant differences were detected in the mean age of both groups $(p>0.05)$. Moreover, informed consent was obtained from all controls and all patients' caregivers. The study was approved by the local ethics committee.

MEGs were recorded with a 148-channel whole-head magnetometer (MAGNES 2500 WH, 4D Neuroimaging), placed in a magnetically shielded room in the "Centro de Magnetoencefalografía Dr. Pérez Modrego". Five minutes of spontaneous MEG activity were acquired at a sampling rate of $678.17 \mathrm{~Hz}$ with subjects in a relaxed state, awake and with eyes closed. A $0.1-200 \mathrm{~Hz}$ hardware bandpass filter and $50 \mathrm{~Hz}$ notch filter were also used. Subsequently, each signal was downsampled by a factor of four to reduce the data length. Artifact-free epochs of length $10 \mathrm{~s}$ (26.4 \pm 5.5 artifact-free epochs per channel and subject, mean \pm SD) were selected for further analysis. Prior to spectral analysis, each segment of 1696 samples was digitally bandpassfiltered with cut-off frequencies at 0.4 and $70 \mathrm{~Hz}$.

\section{B. Definition of the spectral entropies}

The PSD for each MEG segment was calculated from the FT of the autocorrelation function. PSDs were averaged for each channel and subject to compute the mean power spectrum. Then, the RSP was calculated in the classical frequency bands: delta (1-4 Hz), theta $(4-8 \mathrm{~Hz})$, alpha (8-13 $\mathrm{Hz})$, beta1 (13-19 Hz), beta2 (19-30 Hz) and gamma (30-64 $\mathrm{Hz}$ ). If we denote the power at each frequency band as $P_{j}, j$ $=\{$ delta, theta, alpha, beta1, beta 2 , gamma $\}$, then, the RSP can be represented as a probability distribution,

$$
p_{j}=\frac{P_{j}}{\sum_{j} P_{j}} .
$$

Hence, four entropies (SSE, TSE, GSE and RSE) were computed from RSP. The SSE is a disorder quantifier, which has been previously employed to characterize MEG irregularity in $\mathrm{AD}$ [10]. It is a measure of the flatness of the spectrum, and its definition is based on the Shannon's entropy [22],

$$
S S E=-\sum_{j} p_{j} \cdot \ln \left[p_{j}\right] .
$$

The TSE is a generalized information measure, which generalizes the notion of the SSE. As it is a non-logarithmic entropy, it is useful to explore the properties of a probability distribution from a new mathematical framework in relation to the SSE. The TSE is controlled by a parameter $q \in \mathfrak{R}$, which can be considered as measuring the degree of nonextensivity [23]. Hence, it is possible to obtain the Boltzmann-Gibbs entropy from TSE in the limit $q \rightarrow 1$. Its definition is given by [20],

$$
\operatorname{TSE}(q)=\frac{1}{q-1} \sum_{j}\left[p_{j}-\left(p_{j}\right)^{q}\right] .
$$

Regarding to the GSE, it is an entropy closely related to the TSE. Nevertheless, the probability distribution of the original signal is modified to achieve an escort distribution, providing a different framework to analyze the properties of the original signal [24]. Similarly to the TSE, the GSE is also stated by an index $q \in \mathfrak{R}$, which can be identified with the parameter $q$, previously defined for the TSE. Different values of $q$ generate escort distributions of different degree, which can be useful to analyze the mathematical structure of the original distribution. The GSE definition reads [20],

$$
\operatorname{GSE}(q)=\frac{1}{q-1}\left\{1-\left[\sum_{j}\left(p_{j}\right)^{1 / q}\right]^{-q}\right\} .
$$

Finally, the RSE is also a non-extensivity generalized information measure, which can be reduced to the Boltzmann-Gibbs entropy in the limit $q \rightarrow 1$ [25]. This parameter can be used to quantify the uncertainty of a signal, and its definition is given by,

$$
\operatorname{RSE}(q)=\frac{1}{1-q} \ln \left[\sum_{j}\left(p_{j}\right)^{q}\right] .
$$

It should be noticed that in order to obtain a single value per parameter and subject, and to achieve a straightforward interpretation of the results, in this first approach to the problem the measures were averaged for all channels.

All calculations were carried out with the software package Matlab (version 7.0; Mathworks, Natick, MA). 


\section{Statistical analysis}

The Kolmogorov-Smirnov test was used to assess the normal distribution of the variables. After the descriptive analysis, neither several variables nor their log-transformed values met parametric test assumptions. Therefore, nonparametric two-tailed Mann-Whitney U test ( $\alpha=0.05)$ was used to evaluate statistical significance. In addition, binary logistic regression was employed to investigate group classification. Statistical analyses were performed using SPSS software (version 14.0; SPSS Inc, Chicago, Ill).

\section{RESUlTS AND DISCUSSION}

We computed the PSD of the $10 \mathrm{~s}$ segments in the 148 channels. Results were averaged for each channel and each subject. The measures (RSP, SSE, TSE, GSE and RSE) were then computed for each averaged PSD. In order to explore the dependence of the TSE, GSE and RSE on the nonextensive parameter $q$, it was modified $(q=$ $\{1.25,1.5,1.75,2\})$ according to previous studies which analyzed EEG recordings using analogous quantifiers [19], [20]. Finally, mean values for all channels were computed to obtain a quantitative measure per subject. Statistical significance of each parameter was assessed using MannWhitney U tests, whereas classification performance was evaluated by means of binary logistic regression.

Table I summarizes both the mean values of the parameters for each group and the results of statistical and classification analyses. As it can be seen, AD patients exhibit significantly larger $R S P$ values at the delta and theta bands than controls. However, RSP values were significantly lower in $\mathrm{AD}$ patients than in healthy subjects at the beta and gamma bands. This issue implies a slowing of the spontaneous MEG activity in $\mathrm{AD}$, which has been previously reported in similar studies [6]-[11]. Regarding to the spectral entropies, their values indicate a statistically significant decrease in AD patients' MEGs by comparison with healthy subjects. This result suggests an irregularity decrease, in terms of the flatness of the power spectrum, for AD patients. In this sense, previous works obtained similar results, reporting an overall decrease both in irregularity [10] and complexity [12] of MEG activity in AD.

Further inspection of Table I shows that the most significant differences $(p<0.0001)$ were achieved by $R S P$ at the delta and beta bands, with an accuracy between $80.5 \%$ and $85.4 \%$. This issue was also reported by Fernández et al., which performed an exhaustive analysis of the RSP distribution in AD patients' MEGs [8]. With regard to the spectral entropies, the statistical differences achieved with both the $\operatorname{RSE}(\{1.25,1.5,1,75,2\})$ and the $\operatorname{TSE}(1.25)$ were close to those obtained by the $R S P$ at the delta and beta bands. In addition, similar classification results were also obtained by the previous quantifiers, showing an accuracy between $82.9 \%$ and $85.4 \%$. Finally, slightly greater $p$-values than with the previous measures $(p<0.0005)$ were obtained with the $\operatorname{TSE}(\{1.5,1.75,2\}), \quad S S E$ and
$\operatorname{GSE}(\{1.25,1.5,1.75,2\})$, with a maximum accuracy of $82.9 \%$. A similar result was obtained in a previous study ( $p$ $=0.00023 ; 82.93 \%$ accuracy), where the SSE (among other spectral measures) was used to characterize the PSD of MEGs in AD [10].

On the other hand, the analysis of the entropy dependence on the non-extensive parameter $q$, reveals that as $q$ decreases, the TSE, GSE and RSE values approach to those provided by the SSE. Although previous studies have reported that the "background EEG-detection-power" decreases as the parameter $q(q>1)$ increases [17], our results indicate that this issue depends on the quantifier. Hence, the statistical differences observed in the TSE and $G S E$ seem to decrease as $q$ increases, whereas an opposite behavior is obtained when the RSE is analyzed. In addition, previous studies also observed that quantifiers based on Tsallis and Rényi entropies exhibited a moderate or low dependence with the parameter $q$ [18], [20], whereas measures based on the generalized escort-Tsallis statistics showed a strong dependence on $q$ [20]. In our analyses, a slightly dependence on $q$ can also be observed, since both the statistical and classification results varied depending on this index. Nevertheless, further works should be carried out to accurately assess the influence of the non-extensive parameter $q$ on the TSE, GSE and RSE.

In relation to the limitations of the study, it should be mentioned that we have used a small sample size. In this sense, it should also be appropriate to increase the number of subjects enrolled in the work, as well as to extend the analysis to other neurodegenerative disorders with similar alterations to those observed in AD. Finally, as a first approach to the problem we have averaged the whole MEG activity in the head. This issue implies a loss of spatial information and, therefore, further studies should analyze the entropic patterns in each brain region to extend our results.

\section{CONCLUSIONS}

This preliminary study was performed to analyze the spontaneous MEG activity in AD patients and controls by means of several spectral measures. Both a MEG slowing and a loss in irregularity were found using the RSP and the entropic quantifiers, respectively.

Our results suggest that the spectral parameters based on extensive ( $S S E$ ) and non-extensive (TSE, GSE and RSE) informational spectral tools could be useful to characterize the spontaneous MEG activity in $\mathrm{AD}$, leading to a better understanding of the underlying brain dynamics. In this sense, variations of the non-extensive parameter $q$ provide different entropy definitions. They can be used to yield new information about MEG rhythms in AD in comparison with that obtained using conventional spectral methods.

Further works should be performed to accurately explore the influence of the index $q$ and to obtain a detailed spatial analysis. 
TABLE I

MEAN VALUES FOR EACH GROUP (MEAN \pm STANDARD DEVIATION, SD) AND RESUlts OF STATISTICAL AND ClassificATION ANALYSES

\begin{tabular}{|c|c|c|c|c|c|c|c|c|c|}
\hline \multirow{2}{*}{$\frac{\text { Parameter }}{R S P(\text { delta })}$} & \multirow{2}{*}{$\begin{array}{r}\text { Controls } \\
0.20 \pm 0.07\end{array}$} & \multirow{2}{*}{$\frac{\text { AD patients }}{0.38 \pm 0.15}$} & \multirow{2}{*}{$\frac{p \text {-values }}{0.00004}$} & \multicolumn{2}{|c|}{ Sensitivity } & \multicolumn{2}{|c|}{ Specificity } & \multicolumn{2}{|c|}{ Accuracy } \\
\hline & & & & $14 / 20$ & $(70.0 \%)$ & $21 / 21$ & $(100.0 \%)$ & $35 / 41$ & $(85.4 \%)$ \\
\hline$R S P($ theta) & $0.14 \pm 0.07$ & $0.20 \pm 0.10$ & 0.03935 & $10 / 20$ & $(50.0 \%)$ & $18 / 21$ & $(85.7 \%)$ & $28 / 41$ & $(68.3 \%)$ \\
\hline$R S P$ (alpha) & $0.20 \pm 0.08$ & $0.17 \pm 0.08$ & N.S. & $14 / 20$ & $(70.0 \%)$ & $12 / 21$ & $(57.1 \%)$ & $26 / 41$ & $(63.4 \%)$ \\
\hline$R S P($ betal) & $0.17 \pm 0.04$ & $0.10 \pm 0.05$ & 0.00005 & $16 / 20$ & $(80.0 \%)$ & $19 / 21$ & $(90.5 \%)$ & $35 / 41$ & $(85.4 \%)$ \\
\hline$R S P($ beta2) & $0.19 \pm 0.08$ & $0.10 \pm 0.05$ & 0.00008 & $18 / 20$ & $(90.0 \%)$ & $15 / 21$ & $(71.4 \%)$ & $33 / 41$ & $(80.5 \%)$ \\
\hline$R S P$ (gamma) & $0.09 \pm 0.04$ & $0.06 \pm 0.03$ & 0.01891 & $13 / 20$ & $(65.0 \%)$ & $14 / 21$ & $(66.7 \%)$ & $27 / 41$ & $(65.9 \%)$ \\
\hline SSE & $1.66 \pm 0.07$ & $1.46 \pm 0.21$ & 0.00013 & $15 / 20$ & $(75.0 \%)$ & $19 / 21$ & $(90.5 \%)$ & $34 / 41$ & $(82.9 \%)$ \\
\hline$T S E(1.25)$ & $1.34 \pm 0.05$ & $1.18 \pm 0.17$ & 0.00009 & $15 / 20$ & $(75.0 \%)$ & $20 / 21$ & $(95.2 \%)$ & $35 / 41$ & $(85.4 \%)$ \\
\hline$T S E(1.5)$ & $1.10 \pm 0.04$ & $0.97 \pm 0.14$ & 0.00010 & $15 / 20$ & $(75.0 \%)$ & $19 / 21$ & $(90.5 \%)$ & $34 / 41$ & $(82.9 \%)$ \\
\hline $\operatorname{TSE}(1.75)$ & $0.92 \pm 0.03$ & $0.82 \pm 0.11$ & 0.00011 & $15 / 20$ & $(75.0 \%)$ & $19 / 21$ & $(90.5 \%)$ & $34 / 41$ & $(82.9 \%)$ \\
\hline$T S E(2)$ & $0.79 \pm 0.03$ & $0.70 \pm 0.09$ & 0.00011 & $15 / 20$ & $(75.0 \%)$ & $19 / 21$ & $(90.5 \%)$ & $34 / 41$ & $(82.9 \%)$ \\
\hline$G S E(1.25)$ & $1.37 \pm 0.04$ & $1.26 \pm 0.13$ & 0.00019 & $15 / 20$ & $(75.0 \%)$ & $19 / 21$ & $(90.5 \%)$ & $34 / 41$ & $(82.9 \%)$ \\
\hline $\operatorname{GSE}(1.5)$ & $1.14 \pm 0.02$ & $1.08 \pm 0.09$ & 0.00019 & $14 / 20$ & $(70.0 \%)$ & $19 / 21$ & $(90.5 \%)$ & $33 / 41$ & $(80.5 \%)$ \\
\hline$G S E(1.75)$ & $0.96 \pm 0.01$ & $0.92 \pm 0.05$ & 0.00017 & $14 / 20$ & $(70.0 \%)$ & $19 / 21$ & $(90.5 \%)$ & $33 / 41$ & $(80.5 \%)$ \\
\hline$G S E(2)$ & $0.82 \pm 0.01$ & $0.80 \pm 0.03$ & 0.00017 & $14 / 20$ & $(70.0 \%)$ & $19 / 21$ & $(90.5 \%)$ & $33 / 41$ & $(80.5 \%)$ \\
\hline$R S E(1.25)$ & $1.63 \pm 0.08$ & $1.41 \pm 0.23$ & 0.00009 & $14 / 20$ & $(70.0 \%)$ & $20 / 21$ & $(95.2 \%)$ & $34 / 41$ & $(82.9 \%)$ \\
\hline$R S E(1.5)$ & $1.60 \pm 0.09$ & $1.36 \pm 0.25$ & 0.00008 & $16 / 20$ & $(80.0 \%)$ & $19 / 21$ & $(90.5 \%)$ & $35 / 41$ & $(85.4 \%)$ \\
\hline$R S E(1.75)$ & $1.58 \pm 0.10$ & $1.32 \pm 0.26$ & 0.00008 & $16 / 20$ & $(80.0 \%)$ & $19 / 21$ & $(90.5 \%)$ & $35 / 41$ & $(85.4 \%)$ \\
\hline$R S E(2)$ & $1.56 \pm 0.11$ & $1.28 \pm 0.26$ & 0.00007 & $16 / 20$ & $(80.0 \%)$ & $19 / 21$ & $(90.5 \%)$ & $35 / 41$ & $(85.4 \%)$ \\
\hline
\end{tabular}

N.S. = not significant

\section{REFERENCES}

[1] T. D. Bird, "Alzheimer's disease and other primary dementias," in Harrison's Principles of Internal Medicine, E. Braunwald, A. S. Fauci, D. L. Kasper, S. L. Hauser, D. L. Longo, and J. L. Jameson, Eds. New York: McGraw-Hill, 2001, pp. 2391-2399.

[2] M. Rossor, “Alzheimer's disease," in Brain's Diseases of the Nervous System, M. Donaghy, Ed. Oxford: University Press, 2001, pp. 750 754.

[3] R. Hari, "Magnetoencephalography in clinical neurophysiological assessment of human cortical functions," in Electroencephalography: Basic Principles, Clinical Applications, and Related Fields, 5th ed., E. Niedermeyer and F. Lopes da Silva, Eds. Philadelphia: Lippincontt Williams \& Wilkins, 2005, pp. 1165-1197.

[4] J. Jeong, "EEG dynamics in patients with Alzheimer's disease," Clin. Neurophysiol., vol. 115, pp. 1490-1505, 2004.

[5] C. J. Stam, "Nonlinear dynamical analysis of EEG and MEG: review of an emerging field," Clin. Neurophysiol., vol. 116, pp. 2266-2301, 2005 .

[6] H. W. Berendse, J. P. A. Verbunt, Ph. Scheltens, B. W. van Dijk, and E. J. Jonkman, "Magnetoencephalographic analysis of cortical activity in Alzheimer's disease: a pilot study," Clin. Neurophysiol., vol. 111, pp. 604-612, 2000.

[7] D. Osipova, J. Ahveninen, O. Jensen, A. Ylikoski, and E. Pekkonen, "Altered generation of spontaneous oscillations in Alzheimer's disease," NeuroImage, vol. 27, pp. 835-841, 2005.

[8] A. Fernández, R. Hornero, A. Mayo, J. Poza, F. Maestu, and T. Ortiz Alonso, "Quantitative magnetoencephalography of spontaneous brain activity in Alzheimer disease: an exhaustive frequency analysis," Alzheimer Dis. Assoc. Disord., vol. 20, pp. 153-159, 2006.

[9] A. Fernández, R. Hornero, A. Mayo, J. Poza, P. Gil-Gregorio, and T. Ortiz, "MEG spectral profile in Alzheimer's disease and mild cognitive impairment," Clin. Neurophysiol., vol. 117, pp. 306-314, 2006.

[10] J. Poza, R. Hornero, D. Abásolo, A. Fernández, and M. García, "Extraction of spectral based measures from MEG background oscillations in Alzheimer's disease," Med. Eng. Phys., to be published. Available: doi: 10.1016/j.medengphy.2006.11.006.

[11] D. Osipova, K. Rantanen, J. Ahveninen, R. Ylikoski, O. Happola, T. Strandberg, and E. Pekkonen, "Source estimation of spontaneous MEG oscillations in mild cognitive impairment," Neurosci. Lett., vol. 405, pp. 57-61, 2006.

[12] C. Gómez, R. Hornero, D. Abásolo, A. Fernández, and M. López, "Complexity analysis of the magnetoencephalogram background activity in Alzheimer's disease patients," Med. Eng. Phys., vol. 28, pp. $851-859,2006$
[13] A.-M. van Cappellen van Walsum, Y. A. L. Pijnenburg, H. W. Berendse, B. W. van Dijk, D. L. Knol, Ph. Scheltens, and C. J. Stam, "A neural complexity measure applied to MEG data in Alzheimer's disease," Clin. Neurophysiol., vol. 114, pp. 1034-1040, 2003.

[14] R. Franciotti, D. Iacono, S. Della Penna, V. Pizzella, K. Torquati, M. Onofrj, and G. L. Romani, "Cortical rhythms reactivity in AD, LBD and normal subjects. A quantitative MEG study," Neurobiol. Aging, vol. 27 , pp. 1100-1109, 2006

[15] C. J. Stam, A. M. van Cappellen van Walsum, Y. A. L. Pijnenburg, H. W. Berendse, J. C. de Munck, Ph. Scheltens, and B. W. van Dijk, "Generalized synchronization of MEG recordings in Alzheimer's disease: evidence for involvement of the gamma band," J. Clin. Neurophysiol., vol. 19, pp. 562- 574, 2002.

[16] C. J. Stam, B. F. Jones, I. Manshanden, A. M. van Cappellen van Walsum, T. Montez, J. P. Verbunt, J. C. de Munck, B. W. van Dijk, H. W. Berendse, and P. Scheltens, "Magnetoencephalographic evaluation of resting-state functional connectivity in Alzheimer's disease," Neuroimage, vol. 32, pp. 1335-1344, 2006.

[17] A. Capurro, L. Diambra, D. Lorenzo, O. Macadar, M. T. Martin, C. Mostaccio, A. Plastino, J. Pérez, E. Rofman, M. E. Torres, J. Velluti, "Human brain dynamics: the analysis of EEG signals with Tsallis information measure", Physica A, vol. 265, pp. 235-254, 1999.

[18] S. Tong, A. Bezerianos, A. Malhotra, Y. Zhu, and N. Thakor, "Parameterized entropy analysis of EEG following hypoxic-ischemic brain injury," Phys. Lett. A, vol. 314, pp. 354-361, 2003.

[19] N. Kannathal, M. L. Choob, U. R. Acharyab, and P. K. Sadasivana, "Entropies for detection of epilepsy in EEG," Comput. Methods Programs Biomed., vol. 80, pp. 187-194, 2005.

[20] O. A. Rosso, M. T. Martin, A. Figliola, K. Keller, and A. Plastino, "EEG analysis using wavelet-based information tools," J. Neurosci. Methods, vol. 153, pp 163-182, 2006.

[21] G. McKhann, D. Drachman, M. Folstein, R. Katzman, D. Price, E. M. Stadlan, "Clinical diagnosis of Alzheimer's disease: report of NINCDS-ADRDA work group under the auspices of department of health and human services task force on Alzheimer's disease," Neurology, vol. 34, pp. 939-944, 1984.

[22] T. Inouye, K. Shinosaki, H. Sakamoto, S. Toi, S. Ukai, A. Iyama, Y. Katsuda, and M. Hirano, "Quantification of EEG irregularity by use of the entropy of the power spectrum," Electroencephalogr. Clin. Neurophysiol., vol. 79, pp. 204-210, 1991.

[23] C. Tsallis, "Possible generalization of Boltzmann-Gibbs statistics ," $J$. Stat. Phys., vol. 52, pp. 479-487, 1988.

[24] A. Rényi, Probability theory, Amsterdam: North-Holland, 1970.

[25] R. P. Di Sisto, S. Martinez, R. B. Orellana, A. R. Plastino, A. Plastino, "General thermostatistical formalisms, invariance under uniform spectrum translations, and Tsallis q-additivity," Physica A, vol. 590, pp. 590-613, 1999. 